\title{
Esthesioneuroblastoma Masquerading as Chronic Rhinosinusitis
}

\author{
Sylvia G. McCulloch ${ }^{1}$, Harold Y. Lau ${ }^{2}$, Yves Starreveld ${ }^{3}$, \\ John T. Lysack ${ }^{4}$, Desiree Hao ${ }^{2 *}$ \\ ${ }^{1}$ Department of Internal Medicine, Faculty of Medicine, University of Calgary, Calgary, Canada \\ ${ }^{2}$ Tom Baker Cancer Center/University of Calgary, Calgary, Canada \\ ${ }^{3}$ Division of Neurosurgery, Foothills Medical Centre, Calgary, Canada \\ ${ }^{4}$ Clinical Neurosciences, and Surgery, University of Calgary, Department of Diagnostic Imaging, Foothills \\ Medical Centre, Calgary, Canada \\ Email: Sylvia.McCulloch@albertahealthservices.ca, hlau@ucalgary.ca, ystarrev@gmail.com, \\ John.Lysack@albertahealthservices.ca, ${ }^{*}$ Desiree.Hao@albertahealthservices.ca
}

Received 20 June 2015; accepted 4 September 2015; published 7 September 2015

Copyright (C) 2015 by authors and Scientific Research Publishing Inc.

This work is licensed under the Creative Commons Attribution International License (CC BY).

http://creativecommons.org/licenses/by/4.0/

c) (i) Open Access

\begin{abstract}
Background: Esthesioneuroblastoma, a rare tumour of the nasal vault, can present with symptoms that can be mistaken for common conditions such as chronic rhinosinusitis or recurrent epistaxis. Case presentation: A 52-year-old, previously healthy, Caucasian female presented to the emergency room with two months of nasal congestion and a spontaneous, protracted nosebleed that required a nasal clamp to settle. In the following months she had another episode of epistaxis in addition to fatigue, headaches, anosmia, difficulty breathing, worsening vision, weight loss and behavioral changes. Subsequent investigations led to the diagnosis of esthesioneuroblastoma. Conclusion: When patients present with recurrent epistaxis or chronic rhinosinusitis that does not respond to a reasonable course of medical therapy and/or have atypical associated symptoms, further investigations should be considered. The presences of red flags, such as weight loss, severe headaches, vision changes or bizarre behavior are also clues that alternate diagnoses and further additional workup should be considered.
\end{abstract}

\section{Keywords}

Esthesioneuroblastoma, Sinonasal Neoplasms, Sinusitis, Epistaxis

\footnotetext{
*Corresponding author.
}

How to cite this paper: McCulloch, S.G., Lau, H.Y., Starreveld, Y., Lysack, J.T. and Hao, D. (2015) Esthesioneuroblastoma Masquerading as Chronic Rhinosinusitis. Case Reports in Clinical Medicine, 4, 303-308. 


\section{Introduction}

Esthesioneuroblastoma (ENB), also known as olfactory neuroblastoma, is a rare tumour of the nasal vault. The exact incidence is unknown, but there have been more than 1200 cases published in the literature since its discovery in 1924 [1] [2]. Patients with nasal tumours often present with non-specific symptoms that can include unilateral (or bilateral) nasal congestion, epistaxis, and anosmia [1] which can initially be confused with much more common entities, such as chronic rhinosinusitis or recurrent epistaxis [3]. Once sinonasal tumours become more advanced, patients may develop symptoms of local tumour invasion, such as proptosis and excessive lacrimation from orbital involvement, serous otitis media from obstruction of the Eustachian tube, and cranial nerve palsies [3]. The most common site of metastases is the cervical lymph nodes, but a small subset of patients may present with distant spread to bone, lungs, liver, brain or other vital organs [1]. Due to presenting symptoms that overlap with more common conditions, most patients with ENB present with more advanced stage disease, which confers a poorer long-term prognosis [4] [5]. A cohort study by Jethanamest describes the demographics of 311 patients with ENB, the majority of patients are male (55\%), between the ages of 40 to 70 and of Caucasian descent (83\%) [4]. Patients with ENB have differing clinical courses with some living decades with indolent disease and others succumbing quickly to aggressive disease [1] [4]. Due to the rarity of ENB, histopathologic diagnosis can be elusive with ENB mimicking other sinonasal tumors. Correctly differentiating ENB from other malignancies of the sinonasal tract is critical, as the treatment often requires extensive surgical resection with adjunctive radiotherapy, and possibly chemotherapy in advanced cases [5] [7]-[9]. We describe the case of a female who initially presented with epistaxis and nasal congestion but, after the development of additional symptoms, was found to have advanced ENB.

\section{Case Presentation}

A 52-year-old, previously healthy, Caucasian female presented to the emergency room (ER) reporting two months of nasal congestion and presenting with a spontaneous, protracted nosebleed that required a nasal clamp to settle. An X-ray was performed in the ER and antibiotics were prescribed for sinusitis. The patient, who had a history of seasonal allergies, subsequently sought advice from various alternative health care providers for her nasal congestion. The patient and her family understood from the alternative health care providers that the working diagnosis was rhinosinusitis for which she received various alternative treatments for the course of the next ten months. However, in the ensuing months she developed a second episode of epistaxis in addition to fatigue, headaches, anosmia, difficulty breathing, worsening vision and an eleven kilogram weight loss. Her family also noticed a number of behavioral changes. Treatment for her mood was being contemplated while an outpatient referral to Otolaryngology had been initiated. The patient's symptoms persisted and she developed confusion and disorientation which ultimately prompted another visit to the ER. A computed tomography (CT) of the head showed a large soft tissue mass expanding the superior nasal cavity and extending through the skull base into the anterior cranial fossa to cause significant intracranial mass effect (Figure 1). A subsequent MRI showed an aggressive tumour centered on the cribriform plate of the anterior skull base with large intracranial and sinonasal components (Figure 2). A biopsy showed a nest of small blue cells, which was reviewed by a subspecialty pathologist and felt to be consistent with esthesioneuroblastoma. The patient was assessed by at multidisciplinary Head \& Neck Tumour Board rounds. Given that neither a gross total resection nor radical radiation would not have been possible due to bilateral orbit involvement, she was started on chemotherapy in an attempt to downsize the tumour. Unfortunately, the patient's clinical condition deteriorated following her first cycle of chemotherapy and she passed away in the intensive care unit.

\section{Discussion}

A MEDLINE search from 1994-2015 was performed using the keywords "esthesioneuroblastoma" and "olfactory neuroblastoma". Relevant articles that were published in English and limited to humans were included for consideration in the discussion.

Given the non-specific nature of early symptoms, most patients present with advanced stage disease. Data from the Surveillance, Epidemiology and End Results (SEER) tumour registry from 1973-2002 indicates that only $17.2 \%$ of patients with ENB presented with disease limited to the nasal cavity. Of the remaining patients, 49.8\% had tumours involving the nasal cavity and paranasal sinuses, and 3.8\% had tumours extending beyond 


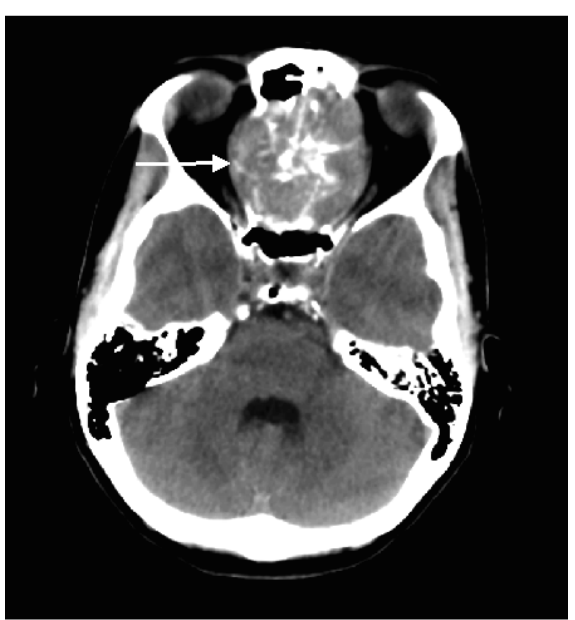

(a)

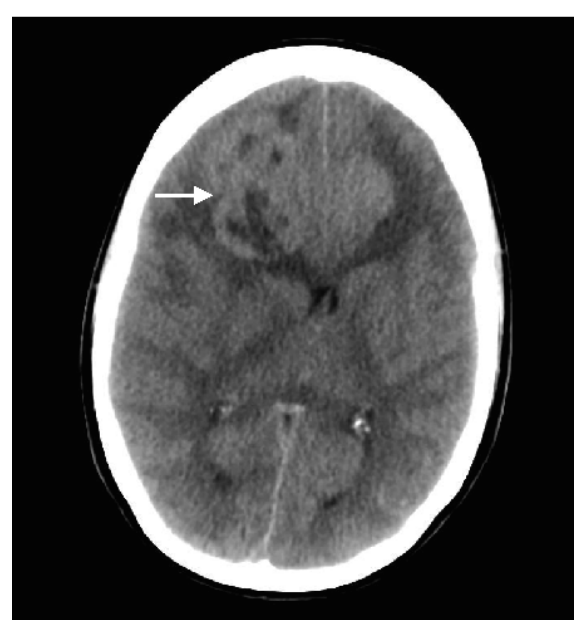

(b)

Figure 1. Noncontrast axial CT images show a large mass centered on the anterior skull base (a) with extension into the anterior cranial fossa (b).

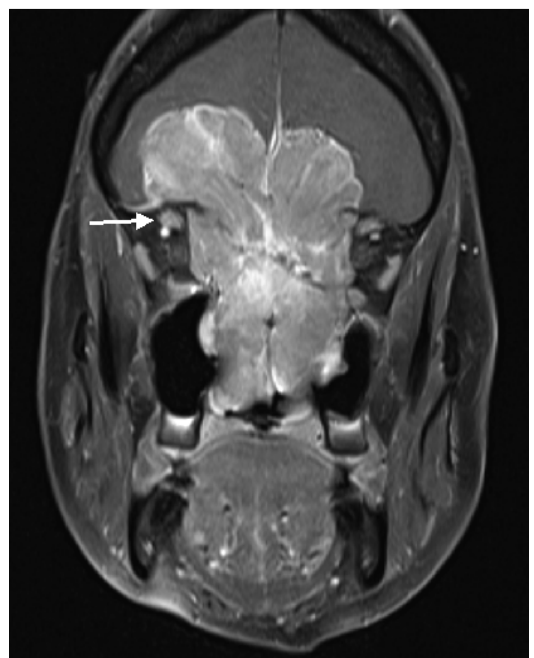

(a)

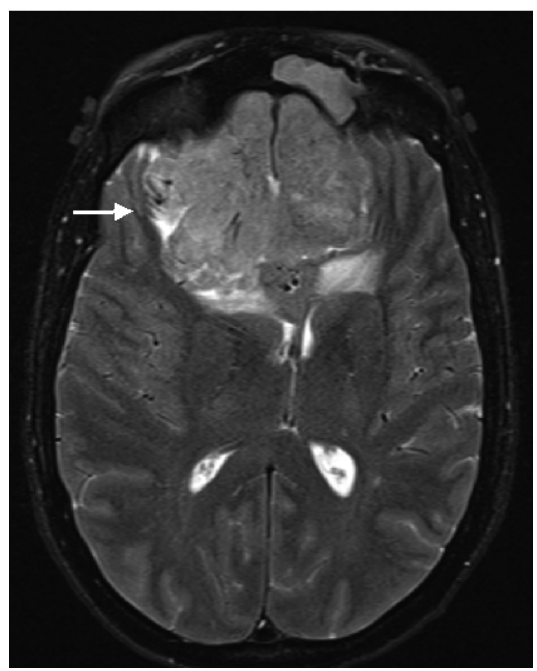

(c)

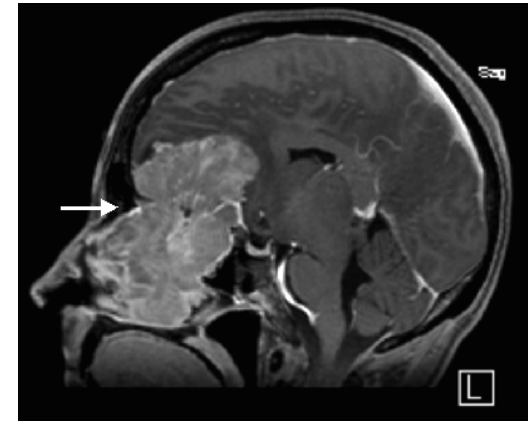

(b)

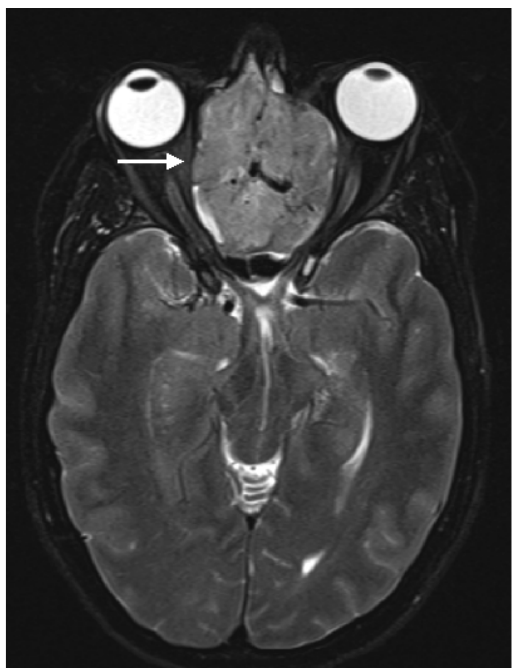

(d) 


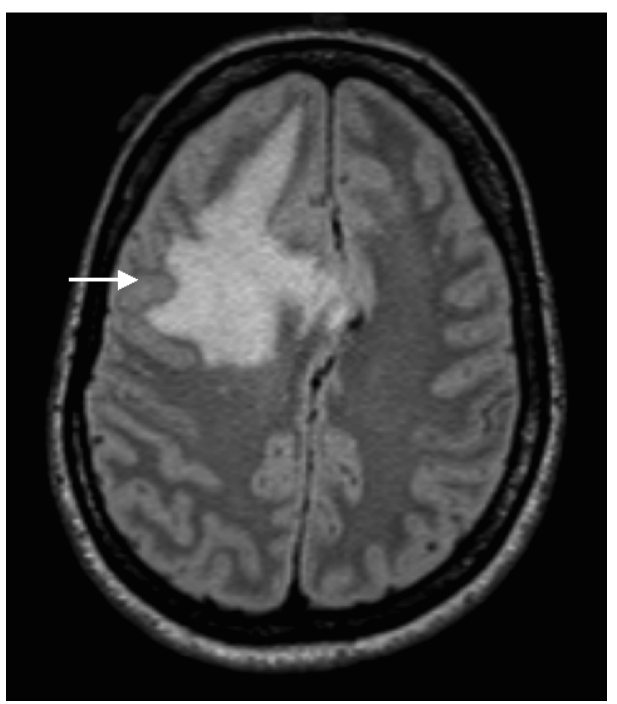

(e)

Figure 2. Postgadolinium T1-weighted MR images in the coronal (a) and sagittal (b) planes show an aggressive tumour centered on the cribriform plate of the anterior skull base with large sinonasal and intracranial components. The coronal image (a) also depicts bilateral intraorbital invasion. Axial T2-weighted images (c, d) show that, despite significant intracranial mass effect, there is no hydrocephalus. However, an axial fluid-attenuated inversion recovery (FLAIR) image (e) demonstrates extensive frontal lobe edema.

those sites. 29.1\% were found to have regional or distant metastases at diagnosis [4]. Less commonly, patients will also present with paraneoplastic syndromes, such as Cushing syndrome or syndrome of inappropriate antidiuretic hormone secretion; the exact incidences of these phenomena are unknown [1] [5].

Findings on plain X-rays of sinonasal tumours are non-specific and often cannot be distinguished from benign conditions, such as nasal polyps [1]. The current standard for imaging ENB includes computed tomography (CT) for locoregional staging and to best evaluate involvement of the surrounding osseous structures. Magnetic resonance imaging (MRI) is complementary and often more accurately determines the margins of the tumour and the extent of invasion of the adjacent soft tissue structures [1] [3]. FDG-PET/CT is also a useful adjunct for determining the extent of local and distant metastases [6]. The gold standard for diagnosis remains histopathological, thus any patients with worrisome imaging studies require referral to a surgeon for biopsy.

Only patients with early stage tumours, located below the cribriform plate, should be treated with single modality therapy, otherwise, data from suggests that surgery followed by radiation in advanced stage tumours affords the greatest chance of cure [5] [7] [8]. Chemotherapy has been used largely for recurrent and metastatic disease, and less commonly for neoadjuvant or adjuvant therapy in conjunction with surgery and/or radiation [9]. Multiple chemotherapy regimens have been reported in case series but, given the rarity of this malignancy, no one regimen has clearly emerged as being optimal [5] [9]-[13]. The prognosis of ENB remains poor with a fiveyear survival of $45 \%$ in most large studies; however for patients with localized disease, who are able to undergo definitive therapy, the 5 year overall survival rate has been reported as high as $63 \%$ - $83.4 \%$ in recent studies [9] [14]. Ow et al. reported a recurrence rate of $46 \%$ in patients who had definitive therapy, with the majority of recurrences occurring regionally. The time to recurrence in this cohort was 6.9 years and median overall survival was 10.5 years [8]. The presence of unresectable disease, metastatic cervical lymphadenopathy and/or distant metastases is the most important prognostic factor and confers a worse overall survival [5] [9].

There are no screening guidelines for ENB or any other nasal tumours. Given that the presenting symptoms of sinonasal tumours overlap to a great extent with common conditions, such as chronic rhinosinusitis, a high level of suspicion is required when patients' present with constitutional symptoms, neurologic symptoms, or other atypical symptoms. Likewise, if patients' symptoms change and/or have not responded to a reasonable trial of conventional medical management for sinusitis, further investigations such as CT and/or a referral to a specialist could help exclude more ominous pathology. 


\section{Conclusion}

In the general population, ENB and other sinonasal tumours are rare. However, when patients present with recurrent epistaxis or chronic rhinosinusitis that does not respond to a reasonable course of medical therapy or have atypical associated symptoms, then further investigations may be required. The presence of red flags, such as weight loss, severe headaches, vision changes or bizarre behavior are also clues that alternate diagnoses and further additional workup should be considered. The initial imaging modality of choice is CT, with MRI subsequently used to complement the CT in positive cases. Plain X-rays are of limited diagnostic utility in this clinical context, and are therefore not recommended.

\section{Acknowledgements}

The authors would like to acknowledge and thank the family of the patient for encouraging the authors to prepare and publish this report.

\section{Consent}

Written informed consent was obtained from the patient's next of kin for publication of this Case report and accompanying images.

\section{Competing Interests}

The authors have no competing interests to disclose.

\section{Authors' Contributions}

SGM performed the literature review and drafted the manuscript; HYL, YS, JL and DH each reviewed and provided multidisciplinary input to edit the manuscript. All authors have read and approved the final manuscript.

\section{References}

[1] Bradley, P.J., Jones, N.S. and Robertson, I. (2003) Diagnosis and Management of Esthesioneuroblastoma. Current Opinion in Otolaryngology \& Head \& Neck Surgery, 11, 112-118. http://dx.doi.org/10.1097/00020840-200304000-00009

[2] Broich, G., Pagliari, A. and Ottaviani, F. (1997) Esthesioneuroblastoma: A General Review of the Cases Published since the Discovery of the Tumour in 1924. Anticancer Research, 17, 2683-2706.

[3] Kairemo, K.J., Jekunen, A.P., Kestila, M.S. and Ramsay, H.A. (1995) Imaging of Olfactory Neuroblastoma-An Analysis of 17 Cases. Auris Nasus Larynx, 25, 173-179. http://dx.doi.org/10.1016/S0385-8146(98)00003-0

[4] Jethanamest, D., Morris, L.G., Sikora, A.G. and Kutler, D.I. (2007) Esthesioneuroblastoma: A Population-Based Analysis of Survival and Prognostic Factors. Archives of Otolaryngology—Head \& Neck Surgery, 133, 276-280. http://dx.doi.org/10.1001/archotol.133.3.276

[5] Dulguerov, P., Allal, A.S. and Calcaterra, T.C. (2001) Esthesioneuroblastoma: A Meta-Analysis and Review. The Lancet Oncology, 2, 683-690. http://dx.doi.org/10.1016/S1470-2045(01)00558-7

[6] Broski, S.M., Hunt, C.H., Johnson, G.B., Subramaniam, R.M. and Peller, P.J. (2012) The Added Value of ${ }^{18}$ F-FDG PET/CT for Evaluation of Patients with Esthesioneuroblastoma. Journal of Nuclear Medicine, 53, 1200-1206. http://dx.doi.org/10.2967/jnumed.112.102897

[7] Benfari, G., Fusconi, M., Ciofalo, A., Gallo, A., Altissimi, G., Celani, T. and De Vincentiis, M. (2008) Radiotherapy Alone for Local Tumor Control in Esthesioneuroblastoma. Acta Otorhinolaryngologica Italica, 28, 292-297.

[8] Ow, T.J., et al. (2014) Optimization of Long Term Outcomes for Patients with Esthesioneuroblastoma. Head \& Neck, 36, 524-530. http://dx.doi.org/10.1002/hed.23327

[9] Modesto, A., et al. (2013) Multimodal Treatment and Long-Term Outcome of Patients with Esthesioneuroblastoma. Oral Oncology, 49, 830-834. http://dx.doi.org/10.1016/j.oraloncology.2013.04.013

[10] Eden, B.V., Debo, R.F., Larner, J.M., Kelly, M.D., Levine, P.A., Stewart, F.M., Cantrell, R.W. and Constable, W.C. (1994) Esthesioneuroblastoma: Long-Term Outcome and Patterns of Failure-The University of Virginia Experience. Cancer, 73, 2556-2562. http://dx.doi.org/10.1002/1097-0142(19940515)73:10<2556::AID-CNCR2820731017>3.0.CO;2-S 
[11] McElroy Jr., E.A., Buckner, J.C. and Lewis, J.E. (1998) Chemotherapy for Advanced Esthesioneuroblastoma: The Mayo Clinic Experience. Neurosurgery, 42, 1023-1027. http://dx.doi.org/10.1097/00006123-199805000-00040

[12] Polin, R., Sheehan, J., Chenelle, A., Munoz, E., Larner, J. and Phillips, C. (1998) The Role of Preoperative Adjuvant Treatment in the Management of Esthesioneuroblastoma: The University of Virginia Experience. Neurosurgery, 42, 1029-1037. http://dx.doi.org/10.1097/00006123-199805000-00045

[13] Bachar, G., Goldstein, D.P., Shah, M., Tandon, A., Ringash, J., Pond, G., Gullane, P.J., Perez-Ordonez, B., Gilbert, R.W., Brown, D.H., Gentili, F., O’Sullivan, B. and Irish, J.C. (2008)Esthesioneuroblastoma: The Princess Margaret Hospital Experience. Head \& Neck, 30, 1607-1614. http://dx.doi.org/10.1002/hed.20920

[14] Rimmer, J., et al. (2014) Olfactory Neuroblastoma: A 35-Year Experience and Suggested Follow-Up Protocol. Laryngoscope, 124, 1542-1549. http://dx.doi.org/10.1002/lary.24562

\section{List of Abbreviations Used}

Computed tomography (CT)

Emergency Room (ER)

Esthesioneuroblastoma (ENB)

Magnetic resonance imaging (MRI)

Surveillance, Epidemiology and End Results (SEER) 